EPJ manuscript No.

(will be inserted by the editor)

\title{
Constraints on the IR behaviour of gluon and ghost propagator from Ward-Slavnov-Taylor identities
}

\author{
Ph. Boucaud ${ }^{1}$, J.P. Leroy ${ }^{1}$, A. Le Yaouanc ${ }^{1}$ A.Y. Lokhov ${ }^{2}$ J. Micheli ${ }^{1}$, O. Pène ${ }^{1}$, J. Rodríguez- \\ Quintero $^{3}$ and C. Roiesnel ${ }^{2}$ \\ $1{ }^{a}$ Laboratoire de Physique Théorique et Hautes Energies; Université de Paris XI, Bâtiment 211, 91405 \\ Orsay Cedex, France \\ 2 Centre de Physique Théorique de l'Ecole Polytechnique; F91128 Palaiseau cedex, France \\ 3 Dpto. Física Aplicada, Fac. Ciencias Experimentales; Universidad de Huelva, 21071 Huelva, Spain.
}

Received: date / Revised version: date

\begin{abstract}
We consider the constraints of the Slavnov-Taylor identity of the IR behaviour of gluon and ghost propagators and their compatibility with solutions of the ghost Dyson-Schwinger equation and with the lattice picture.
\end{abstract}

PACS. PACS-key discribing text of that key - PACS-key discribing text of that key

\section{Introduction}

In ref. 1 we have considered the constraints on the propagator dressing functions which can be derived from the Ward-Slavnov-Taylor identity (WSTI) -supplemented with some minimal assumptions on the analytic behaviour of the former and of the vertex form factors- and we were confronted with a contradiction between them and the ones that stem from the DysonSchwinger equation. The analysis of the ghost propagator Dyson-Schwinger equation seems to indicate that only a non-divergent gluon can match the lattice picture for the infrared behaviour of Landau gauge Green functions. On the other hand, WSTI seems to require that the gluon propagator diverges while the ghost dressing function should be finite and non-vanishing. In that ref. 11 we proposed, as a possible way out, that the ghost-gluon vertex function was singular (which does not contradict Taylor's theorem contrary to frequent claims). That hypothesis did not look very natural and the futher work of [7] made it even less plausible.

In view of the very general validity of the WSTI this situation is rather embarrassing and we wish to reconsider the problem. In the following, we will re-analyse the problem and clarify the working hypotheses to conclude either that the gluon propagator diverges 1 or that some of these hypoteses should fail.

\section{Notations and main hypotheses}

We use the following notations [1:

$$
\begin{aligned}
& \left(F^{(2)}\right)^{a b}\left(k^{2}\right)=-\delta^{a b} \frac{F\left(k^{2}\right)}{k^{2}} \\
& \left(G_{\mu \nu}^{(2)}\right)^{a b}\left(k^{2}\right)=\delta^{a b} \frac{G\left(k^{2}\right)}{k^{2}}\left(\delta_{\mu \nu}-\frac{k_{\mu} k_{\nu}}{k^{2}}\right),
\end{aligned}
$$

${ }^{1}$ Although softly enough as not to contradict the apparent finiteness previously stated from lattice data. 
where $G^{(2)}$ and $F^{(2)}$ are respectively the gluon and ghost propagators, $G$ and $F$ are respectively the gluon and ghost dressing functions. The ghostgluon vertex $\widetilde{\Gamma}_{\mu}(p, k ; q)(k$ and $-p$ are the momenta of the incoming and outgoing ghosts and $q$ the gluon momentum) is defined as follows:

$$
\begin{aligned}
\Gamma_{\mu}^{a b c}(p, k ; q) & =g_{0}\left(-i p_{\nu}\right) f^{a b c} \widetilde{\Gamma}_{\nu \mu}(p, k ; q) \\
& =i g_{0} f^{a b c} \widetilde{\Gamma}_{\mu}(p, k ; q) .
\end{aligned}
$$

It will be also useful to define the following scalars $H_{1}$ and $H_{2}$ :

$\widetilde{\Gamma}_{\mu}(-q, k ; q-k)=q_{\mu} H_{1}(q, k)+(q-k)_{\mu} H_{2}(q, k)$

that, after applying the standard tensor decomposition [5,

$$
\begin{aligned}
\widetilde{\Gamma}_{\nu \mu}(p, k ; q) & =\delta_{\nu \mu} a(p, k ; q)-q_{\nu} k_{\mu} b(p, k ; q) \\
+p_{\nu} q_{\mu} c(p, k ; q) & \left.+q_{\nu} p_{\mu} d(p, k ; q)+p_{\nu} p_{\mu} e(p, k ; q)\right),
\end{aligned}
$$

For our purposes here, we will only assume that the limit of one vanishing gluon momentum can be safely taken, i.e. 2 :

$$
\Gamma_{\lambda \mu \nu}(q-r,-q, r)=\Gamma_{\lambda \mu \nu}(q,-q, 0)+o(1) .
$$

one vanishing gluon momentum while the two others remain finite. Such a limit deserves a careful analysis in the framework of WST identities because of the interplay of gluon and ghost propagator singularities and those of scalar functions in the decomposition [4. When one of the momenta is zero the three-gluon vertex reduces to (cf. ref. [5]):

$$
\begin{aligned}
& \Gamma_{\lambda \mu \nu}(q,-q, 0)= \\
& \left(2 \delta_{\lambda \mu} q_{\nu}-\delta_{\lambda \nu} q_{\mu}-\delta_{\nu \mu} q_{\lambda}\right) T_{1}\left(q^{2}\right) \\
- & \left(\delta_{\lambda \mu}-\frac{q_{\lambda} q_{\mu}}{q^{2}}\right) q_{\nu} T_{2}\left(q^{2}\right)+q_{\lambda} q_{\mu} q_{\nu} T_{3}\left(q^{2}\right) .
\end{aligned}
$$

could be written as follows:

$$
\begin{aligned}
H_{1}(q, k) & =a(-q, k ; q-k)-q^{2}(b(-q, k ; q-k) \\
& +d(-q, k ; q-k)+e(-q, k ; q-k))
\end{aligned}
$$$$
H_{2}(q, k)=q^{2}(b(-q, k ; q-k)-c(-q, k ; q-k))
$$

We can at this point make our first hypothesis: the scalar factors present in that decomposition are regular when one of their arguments goes to zero while the others are kept finite. Thus, we suppose that

$$
a(-r, r-p ; p)=a_{1}\left(p^{2}\right)+\mathcal{O}(p \cdot r),
$$

and the same for the other scalars in the particular kinematic configurations we shall encounter. We adopt the notations: $a_{i}\left(p^{2}\right), b_{i}\left(p^{2}\right), c_{i}\left(p^{2}\right)$ and so on; where the subindex $i$ means that their $i$-th argument is a zero momentum.

The most general tensorial decomposition of the three-gluon vertex, $\Gamma_{\lambda \mu \nu}$ (of course, the antisymmetric color tensor $f^{a b c}$ is factorised) is given in ref. [3. We will be interested in the limit of

\section{WSTI and IR propagators}

The Ward-Slavnov-Taylor ([6]) identity for the three-gluon function reads

$$
\begin{aligned}
p^{\lambda} \Gamma_{\lambda \mu \nu}(p, q, r) & =\frac{F\left(p^{2}\right)}{G\left(r^{2}\right)}\left(\delta_{\lambda \nu} r^{2}-r_{\lambda} r_{\nu}\right) \widetilde{\Gamma}_{\lambda \mu}(r, p ; q) \\
& -\frac{F\left(p^{2}\right)}{G\left(q^{2}\right)}\left(\delta_{\lambda \mu} q^{2}-q_{\lambda} q_{\mu}\right) \widetilde{\Gamma}_{\lambda \nu}(q, p ; r) .
\end{aligned}
$$

We shall now study the behaviour when $r \rightarrow 0$ while keeping $q$ and $p$ finite and apply decompositions (277) and the hypotheses (618) to replace the vertices in Eq. (9). Then, if one only retains

\footnotetext{
${ }^{2}$ It is shown in [3], on a perturbative basis, that the vertex remains finite when one takes the limit $r \rightarrow 0$ while keeping the two other momenta fixed. Our hypothesis amounts to assuming that this result survives beyond perturbation theory.
} 
the leading terms, STI reads

$$
\begin{aligned}
& T_{1}\left(q^{2}\right)\left(q_{\mu} q_{\nu}-q^{2} \delta_{\mu \nu}\right)+q^{2} q_{\mu} q_{\nu} T_{3}\left(q^{2}\right)+ \\
= & \frac{F\left(q^{2}\right)}{G\left(r^{2}\right)}\left[a_{1}\left(q^{2}\right)\left(r^{2} \delta_{\mu \nu}-r_{\mu} r_{\nu}\right)\right. \\
& \left.+b_{1}\left(q^{2}\right) q_{\mu}\left(r^{2} q_{\nu}-(q \cdot r) r_{\nu}\right)+o\left(r^{2}\right)\right] \\
+ & \frac{F\left(q^{2}\right)}{G\left(q^{2}\right)}\left[a_{3}\left(q^{2}\right)\left(q_{\mu} q_{\nu}-q^{2} \delta_{\mu \nu}\right)+o(1)\right] .
\end{aligned}
$$

Thus, if one multiplies both 1.h.s. and r.h.s of this Eq. (10) by $r_{\nu}$, we obtain:

$$
\begin{aligned}
& T_{1}\left(q^{2}\right)\left(q_{\mu}(q \cdot r)-q^{2} r_{\mu}\right) \\
+ & q^{2} q_{\mu}(q \cdot r) T_{3}\left(q^{2}\right)+o(r \cdot q) \\
= & \frac{F\left(q^{2}\right)}{G\left(q^{2}\right)} a_{3}\left(q^{2}\right)\left(q_{\mu}(q \cdot r)-q^{2} r_{\nu}\right)+o(r \cdot q) ;
\end{aligned}
$$

where the first term of r.h.s. of Eq. (10) vanishes because it is transverse to $r_{\nu}$. Thus, by identifying both r.h.s and l.h.s of Eq. (11), one is led to the familiar relations ( $[5])$ :

$$
\begin{aligned}
& T_{1}\left(q^{2}\right)=\frac{F\left(q^{2}\right)}{G\left(q^{2}\right)} a_{3}\left(q^{2}\right) \\
& T_{3}\left(q^{2}\right)=0 .
\end{aligned}
$$

Now, let us multiply both r.h.s and l.h.s. of Eq. (10) by $q_{\mu}$ and apply that $T_{3}$ has been seen to be exactly 0 in Eq. (12) and we obtain then:

$$
\begin{aligned}
& \frac{F\left(q^{2}\right)}{G\left(r^{2}\right)} r^{2}\left[\left(a_{1}\left(q^{2}\right)+q^{2} b_{1}\left(q^{2}\right)\right)\right. \\
\times & \left.\left(q_{\nu}-\frac{(q \cdot r)}{r^{2}} r_{\nu}\right)+o(1)\right]=o(1) .
\end{aligned}
$$

Thus, if $a_{1}\left(q^{2}\right) \neq 0$ or $b_{1}\left(q^{2}\right) \neq 0$ (and, indeed, one knows from perturbation theory that at large momenta $a_{1}=1$, cf. [5, 6] ) (9) implies

$$
\lim _{r \rightarrow 0} \frac{G\left(r^{2}\right)}{r^{2}} \rightarrow \infty
$$

or, in other words, that the gluon propagator diverges in the infrared limit. If we stick to the commonly accepted idea that $\mathrm{G}$ behaves as a power in the infrared $\left(G\left(p^{2}\right) \sim\left(p^{2}\right)^{\alpha_{G}}\right)$ then
$\alpha_{G}<1$ is to be concluded. Another attractive possibility would be to suppose an infrared be(1)haviour less divergent than any power as, for instance, that of the form : $G\left(p^{2}\right) \sim p^{2} \log ^{\nu}\left(p^{2}\right)$ for some positive $\nu$. This will be considered in more detail in a forthcoming paper 4 .

We can also, instead of letting $r \rightarrow 0$, study now the behaviour when $p \rightarrow 0$ of Eq. (9) as is

(10)done in [5]. The dominant part of the l.h.s. of (9]) reads:

$$
\begin{array}{r}
\left(2 \delta_{\mu \nu} p . q-p_{\mu} q_{\nu}-p_{\nu} q_{\mu}\right) a_{3}\left(q^{2}\right) \frac{F\left(q^{2}\right)}{G\left(q^{2}\right)} \\
-\left(\delta_{\mu \nu}-\frac{q_{\mu} q_{\nu}}{q^{2}}\right)(p . q) T_{2}\left(q^{2}\right) ;
\end{array}
$$

where the results in Eq. (12) have been implemented. Let us now multiply both sides with $q^{\mu}$ and keep only the leading tems in $p$ and one obtains :

$$
\begin{aligned}
& \left(q_{\nu}(p \cdot q)-q^{2} p_{\nu}\right) a_{3}\left(q^{2}\right) F\left(q^{2}\right)= \\
& \left(q_{\nu}(p \cdot q)-q^{2} p_{\nu}\right) F\left(p^{2}\right)\left(a_{2}\left(q^{2}\right)-q^{2} d_{2}\left(q^{2}\right)\right) \\
& +\mathcal{O}\left(p^{2}\right)
\end{aligned}
$$

that of course can be true only if $F\left(p^{2}\right)$ goes to some finite limit when $p^{2} \rightarrow 0$ and whence, in terms of scalars,

$$
F\left(q^{2}\right) \underset{q^{2} \rightarrow 0}{\widetilde{T}} F(0) \frac{a_{2}\left(q^{2}\right)-q^{2} d_{2}\left(q^{2}\right)}{a_{3}\left(q^{2}\right)},
$$

where $a_{2} / a_{3} \rightarrow 1$ as $q^{2} \rightarrow 0[5]$.

Let us repeat here that all these considerations are valid only when our regularity hypotheses about the ghost-gluon scalar factors and about the three-gluon vertex (see (68) ) are satisfied. Under those hypotheses one obtains important constraints on the gluon and ghost propagators - namely that they are divergent in the zero momentum limit. Let us now briefly analyze the ghost propagator Dyson-Schwinger equation (GPDSE).

\section{Ghost DSE: the case $\alpha_{F}=0$}

In a previous paper (the first one of ref. [1]) we studied all the classes of solutions for the 
GPDSE, that can be pictured, in diagrammatic form, as:

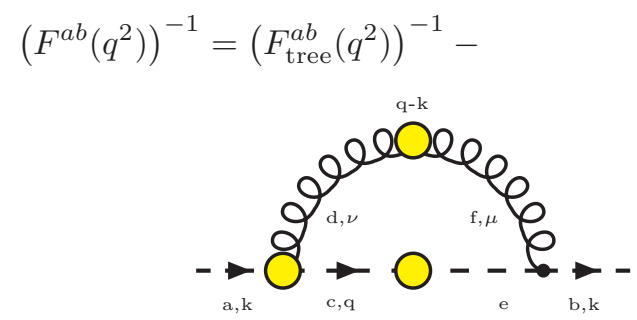

will initially use the common, convenient, but not really justified assumption of a power-law behaviour of the propagators in the deep infrared:

$$
F\left(k^{2}\right) \sim\left(\frac{k^{2}}{q_{0}^{2}}\right)^{\alpha_{F}}, G\left(k^{2}\right) \sim\left(\frac{k^{2}}{q_{0}^{2}}\right)^{\alpha_{G}} .
$$

We shall not repeat here the details of our scaling analysis of Eq. (18) 3 and simply summarize our conclusions in the following 2 tables. It is often claimed, after the study of the GPDSE, that

Let us first recall that the unsubtracted GPDSE $2 \alpha_{F}+\alpha_{G}=0$. In fact, as can be seen in next is actually meaningless since the integral in its tab. 1, this results emerges only 4 after assuming

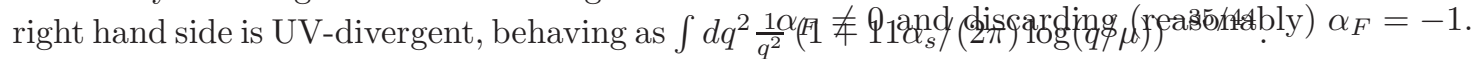
A way out of this difficulty would be to renormalise the equation to deal properly with its UV divergencies. Instead of that, we preferred to study the following subtracted version of bare GPDSE equation for two scales $\lambda k$ and $\kappa \lambda k$ (see Eq.(14) of the first paper quoted in ref. [1]) with $k$ the external ghost momentum and $\kappa$ some fixed number $(<1)$. $\lambda$ is an extra parameter that we shall ultimately let go to 0 in order to study the infared behaviour of the GPDSE. This subtracted version of the GPDSE reads (see Eq.(14) of the first paper quoted in ref. [1]):

$$
\begin{aligned}
& \frac{1}{F(\lambda k)}-\frac{1}{F(\kappa \lambda k)}= \\
& g_{B}^{2} N_{c} \int \frac{d^{4} q}{(2 \pi)^{4}}\left(\frac{F\left(q^{2}\right)}{q^{2}}\left(\frac{(k \cdot q)^{2}}{k^{2}}-q^{2}\right)\right. \\
& \left.\times\left[\frac{G\left((q-\lambda k)^{2}\right) H 1(q, \lambda k)}{(q-\lambda k)^{2}}-(\lambda \rightarrow \kappa \lambda)\right]\right)
\end{aligned}
$$

where $H_{1}$ is the particular combination of the scalars defined in Eq. (2) playing the GPSDE game. Furthermore, a proper dimensional analysis of Eq. (18) requires to cut the integration domain in its r.h.s. into two pieces by introducing some additional scale $q_{0}^{2}$ (of the order of $\Lambda_{\mathrm{QCD}}^{2}$ ). Clearly the external momentum is not the only relevant scale in the problem and $\Lambda_{\mathrm{QCD}}$, without which it would not be understandable that the UV behaviour differs drastically from the IR one, must be taken into account. A careful dimensional analysis of the integrals extended over both domains, $q^{2}>q_{0}^{2}$ and $q^{2}<q_{0}^{2}$, is mandatory [1. In the second one -and only there- we

\begin{tabular}{|c|c|c|}
\multicolumn{3}{|c}{$\alpha_{F} \neq 0$} \\
\hline$\alpha_{F}+\alpha_{G}$ & r.h.s. & constraint \\
\hline$>1$ & $\lambda^{2}$ & $\alpha_{F}=-1$ \\
\hline$=1$ & $\lambda^{2} \log \lambda$ & excluded \\
\hline$<1$ & $\left(\lambda^{2}\right)^{\alpha_{F}+\alpha_{G}}$ & $2 \alpha_{F}+\alpha_{G}=0$ \\
\hline
\end{tabular}

Table 1. Constraints imposed by the GPDSE to the critical behaviour of ghost and gluon propagators for the case $\alpha_{F} \neq 0$. The second column shows the behaviour on $\lambda(\lambda \rightarrow 0)$ of Eq. (18)'s r.h.s., while l.h.s. behaves as $\left(\lambda^{2}\right)^{-\alpha_{F}}$.

However, if $\alpha_{F}=0$ another solutions are also compatible with GPDSE (see tab. 2).

Some recent lattice results seem to exclude the standard $\left(2 \alpha_{G}+\alpha_{F}=0\right)$-solution [1,7]. If one admits these results (lattice also discards $\alpha_{F}=-1$ ), then one is led to conclude that GPDSE implies $\alpha_{F}=0$.

Furthermore, it was shown in ref. 2 that the r.h.s. of Eq. (18) is the sum of two terms behaving respectively as $\lambda^{2 \operatorname{Min}\left(\alpha_{F}+\alpha_{G}+\alpha_{\Gamma}, 1\right)}$ and $\lambda^{2}$ when $\lambda \rightarrow 0$. So it behaves as $\lambda^{2}$ when $\alpha_{F}=0$. Then, one can proves that for any $\kappa$ there is a

3 The analysis done in 1 missed some possible solutions (for instance, the case $\alpha_{F}=0, \alpha_{G}<1$ ) mainly because of the fact that we had rejected the possibility of non-analytic sub-dominant terms in the dressing functions

4 The regularity of the ghost-gluon vertex is also needed as was discussed in 1 . 


\begin{tabular}{|c|c|c|}
\multicolumn{3}{|c}{$\alpha_{F}=0$} \\
\hline$\alpha_{F}+\alpha_{G}$ & r.h.s. & constraint \\
\hline$>1$ & $\lambda^{2}$ & $F\left(q^{2}\right)=A+B q^{2}$ \\
\hline$=1$ & $\lambda^{2} \log \lambda$ & $F\left(q^{2}\right)=A+B q^{2} \log q^{2}$ \\
\hline$<1$ & $\left(\lambda^{2}\right)^{\alpha_{G}}$ & $F\left(q^{2}\right)=A+B q^{2 \alpha_{G}}$ \\
\hline
\end{tabular}

Table 2. The same constraints analysed in tab. 1 but here for $\alpha_{F}=0$. The 1.h.s. of Eq. (18) behaves now as the next-to-leading term of the deep infrared expansion of $F\left(q^{2}\right)$ (third column).

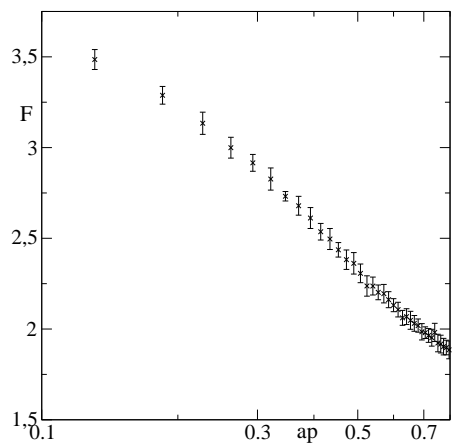

Fig. 1. F(p) from a $\mathrm{SU}(2)$ simulations on a $48^{4}$ lattice at $\beta=2.3$

value of $\lambda$ and $c$ such that

$$
\left|\frac{1}{F(\lambda k)}-\frac{1}{F\left(\kappa^{n} \lambda k\right)}\right| \leq c \frac{1-\kappa^{2 n}}{1-\kappa^{2}} \lambda^{2} .
$$

So $F \rightarrow \infty$ when $\lambda \rightarrow 0$ is excluded because taking the limit of the above expression when $n \rightarrow \infty$ we should have $\left|\frac{1}{F(\lambda k)}\right| \leq c \frac{1}{1-\kappa^{2}} \lambda^{2}$ and $\mathrm{F}$ would diverge as or more rapidly than $\frac{1}{\lambda^{2}} \mathrm{im}$ plying $\alpha_{F} \leq-1$ in contradiction with the hypothesis $\alpha_{F}=0$. Let us remark that $F \rightarrow 0$ is also excluded: Eq. (20) implies $\left|\frac{1}{F\left(\kappa^{n} \lambda k\right)}\right| \leq$ $\left|\frac{1}{F(\lambda k)}\right|+c \frac{1-\kappa^{2 n}}{1-\kappa^{2}} \lambda^{2}$ and $\frac{1}{F\left(\kappa^{n} \lambda k\right)}$ cannot tend to infinity when $n \rightarrow \infty$. It should be emphasized that the dimensional analysis driving to Eq. (20) is also valid if $F\left(q^{2}\right)$ is admitted to behave in a way other than a power. Thus, if a leading power behaviour is discarded for the ghost dressing function, it has to be finite and $\neq 0$ in the IR limit.

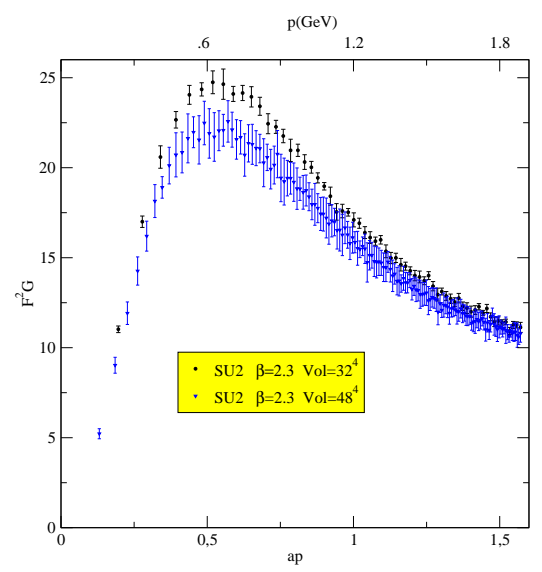

Fig. 2. $F^{2} G$ from lattice simulation for $S U(2)\left(32^{4}\right.$ and $\left.48^{4}, \beta_{S U(2)}=2.3\right)$ gauge groups. $2 \alpha_{F}+\alpha_{G}=0$ implies a constant in the infrared domain.

\section{5 conclusion}

We derive from the Ward-Slavnov-Taylor identity for the three-gluon and ghost-gluon vertices, after assuming their regularity that gluon propagator diverges and ghost dressing function remains finite as the momentum goes to zero. A dimensional analysis of the GPDSE, provided that we trust the lattice results excluding $2 \alpha_{F}+\alpha_{G}=$ 0 [1,7] and $\alpha_{F}=-1$, leads to conclude independently that the ghost dressing function remains finite at zero momentum [2] (see tabs. 112). Both GPDSE and WSTI constraints will offer compatible solutions provided that one admits nonanalytic sub-leading terms for the low momentum expansion of dressing functions.

On the other hand, such a solution respecting WSTI and GPDSE constraints still match in the present picture of lattice knowledge about the IR behavior of propagators and vertices. The current simulations of ghost-gluon vertex seems to discard $2 \alpha_{F}+\alpha_{G}=0$ but those of ghost and gluon propagators cannot yet exclude or confirm the smooth divergences we propose as a way out 7, 8, 9] (as an example, see Fig. 11from ref. [2] or Fig. 2 from ref. 11). A non-power behaviour (logarithmic, for instance) could be specially elusive for lattice extrapolations at infinite volume. Of course, new simulation results on bigger lat- 
tice volumes (or with twisted boundary conditions [11) and careful extrapolations will be very welcome to dig into this matter.

This is a very interesting task to be acomplished, because either such a logarithmic (or similar) behaviour is found or one is led to conclude that the tensorial decomposition of ghostgluon or three-gluon vertex admits non-regularities.

\section{References}

1. Ph. Boucaud et al., arXiv:hep-ph/0507104;

2. Ph. Boucaud et al., JHEP 0606 (2006) 001

3. J. S. Ball, T. W. Chiu, Phys. Rev. D 22, 2550 (1980).

4. Ph. Boucaud et al., in progress.

5. K. G. Chetyrkin and A. Retey, arXiv:hep-ph/0007088; A. I. Davydychev, P. Osland and O. V. Tarasov, Phys. Rev. D 54, 4087 (1996) [Erratum-ibid. D 59, 109901 (1999)]

6. J. C. Taylor, Nucl. Phys. B 33, 436-444 (1971);

A. A. Slavnov, Theor. Math. Phys. 10 (1972) 99

7. A. Sternbeck, E. M. Ilgenfritz, M. MullerPreussker and A. Schiller, Nucl. Phys. Proc. Suppl. 153 (2006) 185

8. F. D. R. Bonnet, P. O. Bowman, D. B. Leinweber, A. G. Williams, J. M. Zanotti, Phys. Rev. D 64 (2001) 034501

9. D. Becirevic, P. Boucaud, J. P. Leroy, J. Micheli, O. Pene, J. Rodriguez-Quintero and C. Roiesnel, Phys. Rev. D 61 (2000) 114508

10. P. J. Silva and O. Oliveira, Phys. Rev. D 74 (2006) 034513

11. T. Tok, K. Langfeld, H. Reinhardt and L. von Smekal, "The gluon propagator in lattice Landau gauge with twisted boundary PoS LAT2005 (2006) 334 arXiv:hep-lat/0509134. 
